\title{
Avaliação da dinâmica litorânea da região de Baixio/Barra do Itariri, litoral norte do Estado da Bahia, utilizando o Sistema de Modelagem Costeira (SMC-Brasil)
}

\author{
Iracema Reimão SILVA ${ }^{1}$, Júnia Kacenelenbogen GUIMARÃES ${ }^{2}$, Abílio Carlos da Silva Pinto BIT- \\ TENCOURT $^{3}$, Tais Kalil RODRIGUES ${ }^{4}$ \& Gerson FERNANDINO de Andrade Neto ${ }^{5}$
}

1 Departamento de Oceanografia, Instituto de Geociências, Universidade Federal da Bahia. Rua Barão de Jeremoabo, s/n, Campus Universitário de Ondina, CEP. 40.170-020, Salvador, BA, Brasil (iracema@pq.cnpq.br).

2 Departamento de Geografia, Instituto de Geociências, Universidade Federal da Bahia. Rua Barão de Jeremoabo, s/n, Campus Universitário de Ondina, CEP 40.170-020, Salvador, BA, Brasil (jkg@ufba.br).

3 Laboratório de Estudos Costeiros, Instituto de Geociências, Universidade Federal da Bahia. Rua Barão de Jeremoabo, s/n, Campus Universitário de Ondina, CEP 40.170-020, Salvador, BA, Brasil (abilio@pq.cnpq.br).

4 Departamento de Geografia, Universidade Federal da Sergipe. Cidade Universitária Prof. José Aloísio de Campos, Av. Marechal Rondon, s/n Jardim Rosa Elze, CEP 49100-000, São Cristóvão, SE, Brasil (tkalilr@yahoo.com.br).

5 Curso de Pós-Graduação em Geologia, Instituto de Geociências, Universidade Federal da Bahia. Rua Barão de Jeremoabo, s/n, Campus Universitário de Ondina, CEP 40.170-020, Salvador, BA, Brasil (gerson.fernandino@yahoo.com.br).

Recebido em 07/2016. Aceito para publicação em 07/2017.

Versão online publicada em 21/10/2017 (www.pesquisasemgeociencias.ufrgs.br)

\begin{abstract}
Resumo - O Sistema de Modelagem Costeira (SMC-Brasil) integra diferentes modelos numéricos que permitem realizar análises em curto, médio e longo prazo de uma praia, contribuindo para estudos e planos de gestão litorâneos. 0 objetivo deste trabalho é caracterizar a dinâmica de ondas e correntes costeiras do trecho litorâneo entre os povoados de Baixio e Barra do Itariri, litoral norte do Estado da Bahia, a fim de gerar modelos de propagação de ondas, transporte, fluxo sedimentar e cota de inundação. A análise estatística de ondas para a área de estudo, em termos de condições médias e extremas, realizada para águas intermediárias, no Ponto 1 (Ponto Dow), indicou um predomínio das ondas vindas de ESE e SE, com alturas variando de 1,61 a 3,25 m e períodos variando de 7,87 a 13,81 s. A partir deste ponto foi realizada a modelagem do clima de ondas em águas rasas, analisada em outros sete pontos do litoral. Esta análise indicou uma predominância das ondas de SE, alcançando alturas em torno de 1,4 m na zona de arrebentação, e a ocorrência de três zonas de correntes de retorno. 0 cálculo das taxas médias anuais de transporte longitudinal mostrou a existência de uma zona de divergência no sentido do transporte líquido nas proximidades de Baixio e um aumento no percentual de incidência das ondas de SE seguindo para norte da área. A combinação de eventos de tempestades com marés meteorológicas positivas, marés astronômicas de sizígia e valores extremos de espraiamento de onda, pode resultar em situações de alto risco para ecossistemas e propriedades, com grandes impactos para o litoral estudado, que atualmente já apresenta uma alta vulnerabilidade à erosão costeira.
\end{abstract}

Palavras-chave: erosão costeira, transporte sedimentar, clima de ondas, cota de inundação.

\begin{abstract}
EVALUATION OF THE COASTAL DYNAMICS OF THE AREA OF BAIXIO/BARRA DO ITARIRI, NORTHERN COAST of the State of Bahia, using the Sistema de Modelagem Costeira (SMC-Brasil). The software Sistema de Modelagem Costeira (SMC-Brasil) performs various numerical models that allow for conducting short-, middle- and long-term analyses on beaches, thus contributing to coastal studies and management plans. The objective of the present study was to characterize wave and coastal current dynamics of the coastal area of the settlings of Baixio and Barra do Itariri, northern coast of the State of Bahia, producing wave propagation, sediment transport and flux, and inundation level. The wave statistical analysis for the study area, in terms of mean and extreme conditions, performed for intermediate waters, in Point 1 (Dow Point), indicated a predominance of waves from ESE and SE, with heights ranging between 1.61 and $3.25 \mathrm{~m}$ and periods, between 7.87 and $13.81 \mathrm{~s}$. Shallow-water wave climate modelling was performed based on Point 1, analyzing others seven profiles along the coast. This analysis indicated a predominance of waves from SE, reaching approximately $1.4 \mathrm{~m}$ of height in the surf zone, and the occurrence of three rip currents. The calculation of mean annual longshore transport rates indicated the existence of a divergence zone in the direction of the net transport near Baixio and a northward increase in the percentage of incidence of waves from SE. The combination of storm events with positive meteorological tides, astronomical spring tides and extreme run-up values, can result in high-risk situations for ecosystems and properties, with great impacts on the studied coast, which currently presents high vulnerability to coastal erosion.
\end{abstract}

Keywords: coastal erosion, sediment transport, wave climate, inundation level. 


\section{Introdução}

Algumas características geomorfológicas e hidrodinâmicas das praias podem representar uma alta vulnerabilidade a riscos naturais (Calliari et al., 2010) associadas, por exemplo, a eventos extremos. Estudos neste sentido tornam-se cada vez mais necessários frente às incertezas relacionadas às mudanças climáticas. A possibilidade de ocorrência de alterações climáticas, com maior incidência de eventos extremos (Douglas \& Peltier, 2002; Tronis, 2004; Walsh et al., 2004; IPCC, 2007; Marengo, 2008; Diniz \& Resende, 2014), ainda que não seja consenso científico (Molion, 2008; Oliveira \& Vecchia, 2009; Casagrande et al., 2011; Miranda \& Araújo, 2014), pode representar uma grave ameaça ambiental e social, especialmente para os municípios litorâneos.

Além disso, mudanças climáticas em escalas de tempo de décadas e de séculos podem produzir mudanças ecológicas significativas (Dominguez \& Bittencourt, 1994) capazes de gerar graves impactos à vida humana e ao meio ambiente. Exemplos são a intrusão de água salgada em águas superficiais e subterrâneas (Muehe \& Neves, 1995; Fitzgerald et al., 2008) e o declínio da área ocupada por marismas e manguezais (Muehe \& Neves, 1995; Nicholls \& Leatherman, 1995; Nicholls \& Cazenave, 2010).

A vulnerabilidade ambiental do litoral brasileiro é agravada pelo seu padrão de ocupação e pela falta de planejamento territorial na maior parte de suas cidades costeiras (Ribeiro, 2008). Segundo Pereira \& Coelho (2013), a transformação do potencial de vulnerabilidade, ou de perigo natural, para uma situação de risco depende da presença de pessoas ou construções. Assim, risco à vida ou às propriedades expressa a dimensão social dos eventos e a percepção individual dos seus efeitos (Egler, 1996).

O Sistema de Modelagem Costeira (SMC-Brasil) tenta cobrir uma lacuna na disponibilização de dados de clima de ondas em séries temporais longas e contínuas para o litoral brasileiro (Almeida et al., 2015), disponibilizando em seu banco de dados uma série temporal de 60 anos, de 1948 a 2008, com resolução horária (Camus et al., 2013). O SMC-Brasil foi desenvolvido por pesquisadores do Instituto Hidráulico Ambiental da Cantábria (IH Cantábria), da Universidade da Cantábria (Santander, Espanha) e, posteriormente, adaptado e transferido para a administração pública brasileira. Trata-se de uma ferramenta computacional que combina metodologias de trabalho, bases de dados de cartas náuticas e modelos numéricos orienta- dos para o estudo e sugestão de soluções de problemas em zonas costeiras. Assim, a partir do SMC-Brasil, podem ser executados diferentes modelos numéricos, que permitem realizar análises em escalas de curto, médio e longo prazo em relação ao comportamento de uma praia (Camus et al., 2013).

A utilização do SMC-Brasil no presente trabalho representa uma oportunidade de ratificação e teste desta ferramenta para estudos no litoral brasileiro, demonstrando sua aplicação como ferramenta para a gestão costeira, com a geração de modelos que consideram a série temporal ampla (60 anos), acima mencionada. Um dos estudos piloto do SMC-Brasil foi desenvolvido por Luca (2011), testando esta ferramenta numérica e seu banco de dados para a Praia de Massaguaçu, litoral de São Paulo. Almeida (2013) aplicou esta ferramenta para estudar a dinâmica litorânea e a evolução da zona costeira da Praia de Piçarras, no litoral de Santa Catarina. Rodrigues (2014) utilizou o SMC-Brasil para modelar o clima de ondas e o transporte sedimentar das praias de Aracaju. No litoral do Rio Grande do Norte, na Praia de Ponta Negra, Almeida et al. (2015) aplicaram o SMC-Brasil para a modelagem do clima de ondas local, contribuindo para a gestão e planejamento de uso desta praia.

O litoral norte do Estado da Bahia abrange os municípios de Lauro de Freitas, Camaçari, Mata do São João, Entre Rios, Esplanada, Conde e Jandaíra (Fig. 1). Ainda que a ocupação da linha de costa no município de Esplanada, onde está localizada a Praia de Baixio (Fig. 1), seja bastante rarefeita (Silva et al., 2012), este estudo se justifica pela crescente ocupação da sua zona costeira por grandes complexos hoteleiros. A beleza natural da Praia de Baixio e de sua zona costeira, com terraços arenosos, rios, dunas, manguezais e diversas lagoas, têm atraído cada vez mais os investimentos imobiliários e turísticos. Localizada a cerca de $120 \mathrm{~km}$ de Salvador e com acesso asfaltado, esta praia receberá um grande complexo hoteleiro, atualmente em processo de implantação. A ocupação deste trecho costeiro, próximo a uma desembocadura fluvial e localizado em uma zona de divergência no sentido do transporte litorâneo de sedimentos (Livramento, 2008; Bittencourt et al., 2010) (Fig. 2), pode acelerar os processos erosivos na região, dificultando o livre recuo da faixa arenosa e favorecendo a ação de reflexão das ondas em construções de alvenaria.

Nesse contexto, o objetivo deste trabalho é caracterizar a dinâmica de ondas e correntes costeiras do trecho litorâneo entre os povoados de Baixio e de Barra do Itariri, no litoral norte do estado da Bahia. Modelos de propagação de ondas, de trans- 
porte, de fluxo sedimentar e da cota de inundação foram gerados, na tentativa de contribuir para a gestão deste litoral.

\section{2 Área, materiais e métodos}

\subsection{Localização da área}

No contexto regional, a área de estudo entre Baixio e Barra do Itariri, está inserida no litoral norte do estado da Bahia (Fig. 1), o qual apresenta uma ocupação urbana concentrada nos três municípios mais próximos à região metropolitana de Salvador - Lauro de Freitas, Camaçari e Mata de
São João - e uma ocupação mais rarefeita nos demais municípios.

Em termos geológicos, a zona costeira (Fig. 2) é delimitada, na parte interna, por depósitos semiconsolidados da Formação Barreiras (Martin et al., 1980a; Dominguez et al., 2009), de idade miocênica (Suguio \& Nogueira, 1999). Depósitos quaternários, representados fundamentalmente por terraços marinhos arenosos pleistocênicos e holocênicos são encontrados em quase toda a extensão dessa região costeira (Martin et al., 1980a). Em alguns locais ocorrem depósitos flúvio-lagunares de terras úmidas, lagoas (Fig. 2), manguezais e dunas (Martin et al., 1980a; Leão \& Kikuchi 1999).

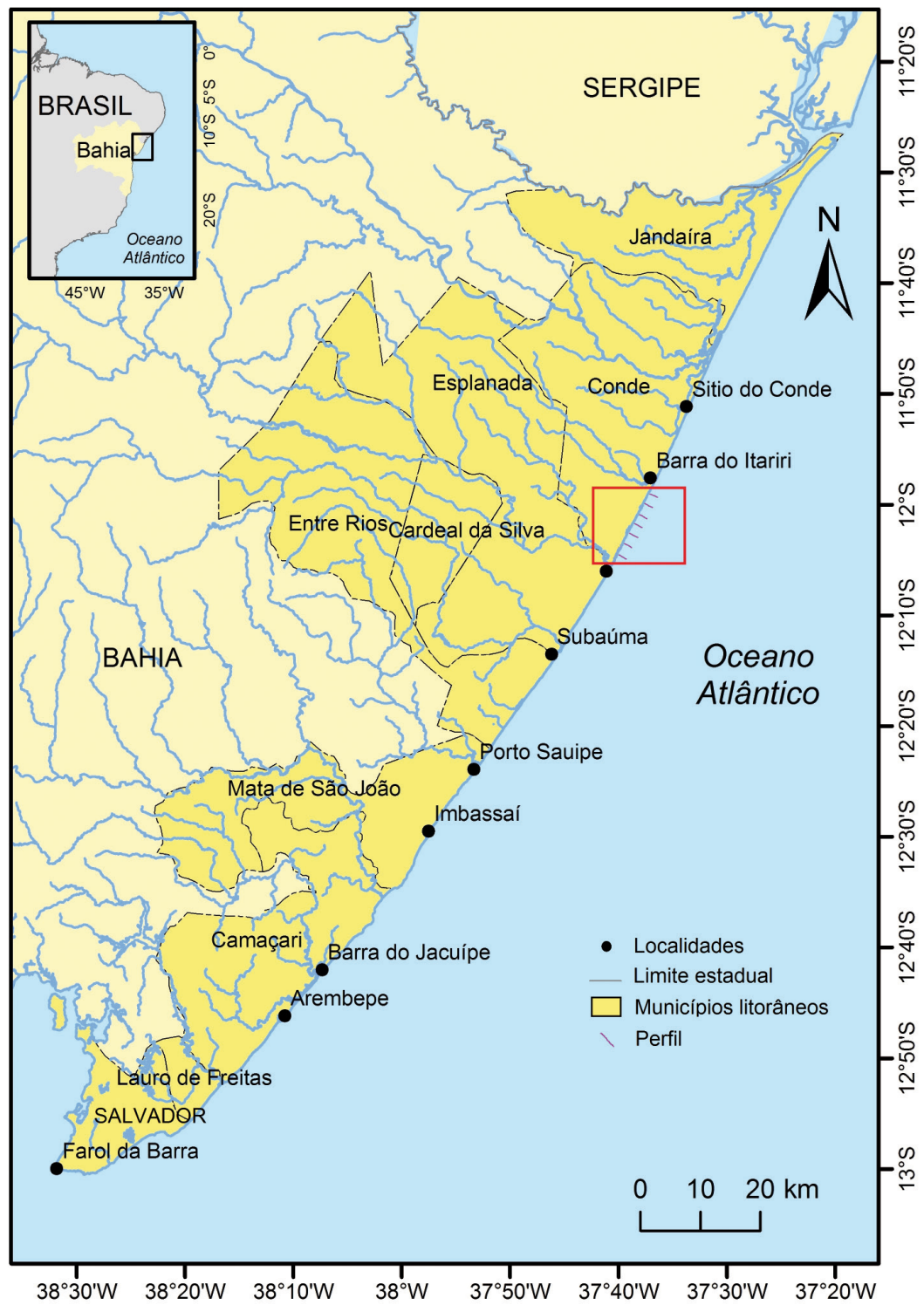

Figura 1. Localização da área de estudo, Praia de Baixio (Município de Esplanada), litoral norte do Estado da Bahia. Figure 1. Study area location, Baixio Beach (Municipality of Esplanada), northern coast of the State of Bahia. 


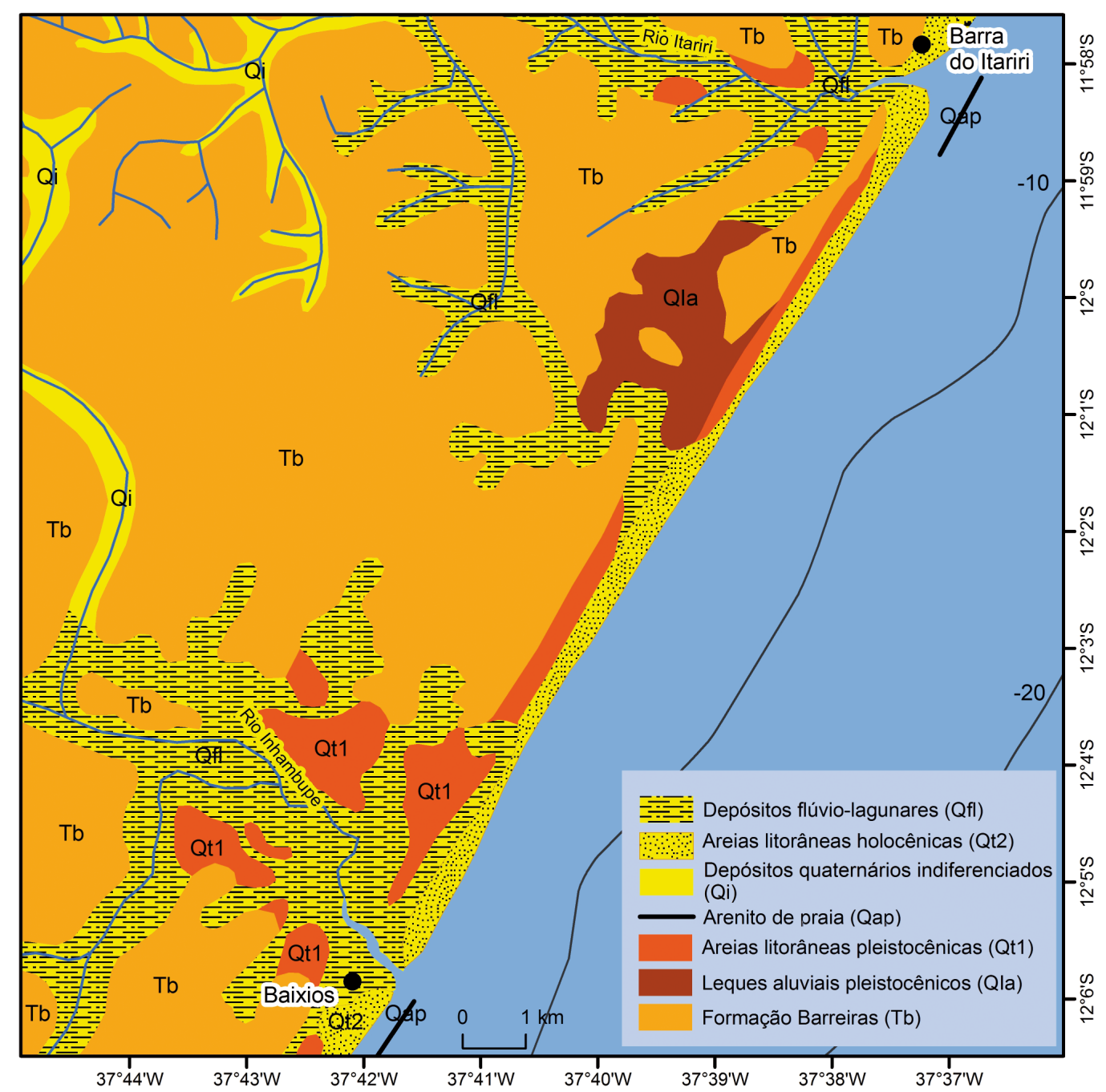

Figura 2. Principais unidades geológicas da área de estudo (Fonte: Martin et al., 1980b).

Figure 2. Main geological units of the study area (Source: Martin et al., 1980b).

A linha de costa, com cerca de $10 \mathrm{~km}$ de extensão e constituída de praias arenosas predominantemente desertas, apresenta um contorno retilíneo bastante regular. É bordejada, na sua maior parte, por um cordão-duna (Fig. 3) com altura média de $2 \mathrm{~m}$, apresentando uma escarpa erosiva não sazonal, constituída por areias finas que são parcialmente retidas pela vegetação herbácea de restinga adjacente à linha de preamar máxima de sizígia (Bittencourt et al., 2010; Santana Neto, 2012).

Conforme o modelo proposto por Wright \& Short (1984), quanto à morfodinâmica praial, a praia da área de estudo foi classificada como intermediária (Livramento, 2013), composta por sedimentos de tamanho areia média, e composição quartzosa, com declividades variando de 5 a $10^{\circ}$ e larguras entre 25 e 60 m (Livramento, 2013). É comum a presença de bancos e cavas paralelos à linha de costa, algumas vezes expostos durante pe- ríodos de maré baixa (Santana Neto, 2012).

A zona de divergência, como mencionado anteriormente, com sentido da deriva litorânea de sul para norte entre Baixio e Barra do Itariri e, de norte para sul, entre Baixio e Subaúma (Fig. 1), segundo Livramento (2008) e Bittencourt et al. (2010) é a principal responsável pela ocorrência de erosão no cordão-duna presente neste trecho costeiro. Santana Neto (2012), além de confirmar a existência desta zona de divergência no sentido da deriva litorânea, indicou que o predomínio das ondas de sudeste e sul-sudeste, com maiores alturas e, portanto, maior competência de transporte (com sentido aproximado de SW para NE), entre Baixio e Barra do Itariri, pode ser um fator determinante para a maior ocorrência de lixo marinho nesse trecho - quando comparado com o trecho mais ao sul, entre Subaúma e Baixio - especialmente durante o período de outono/inverno. 


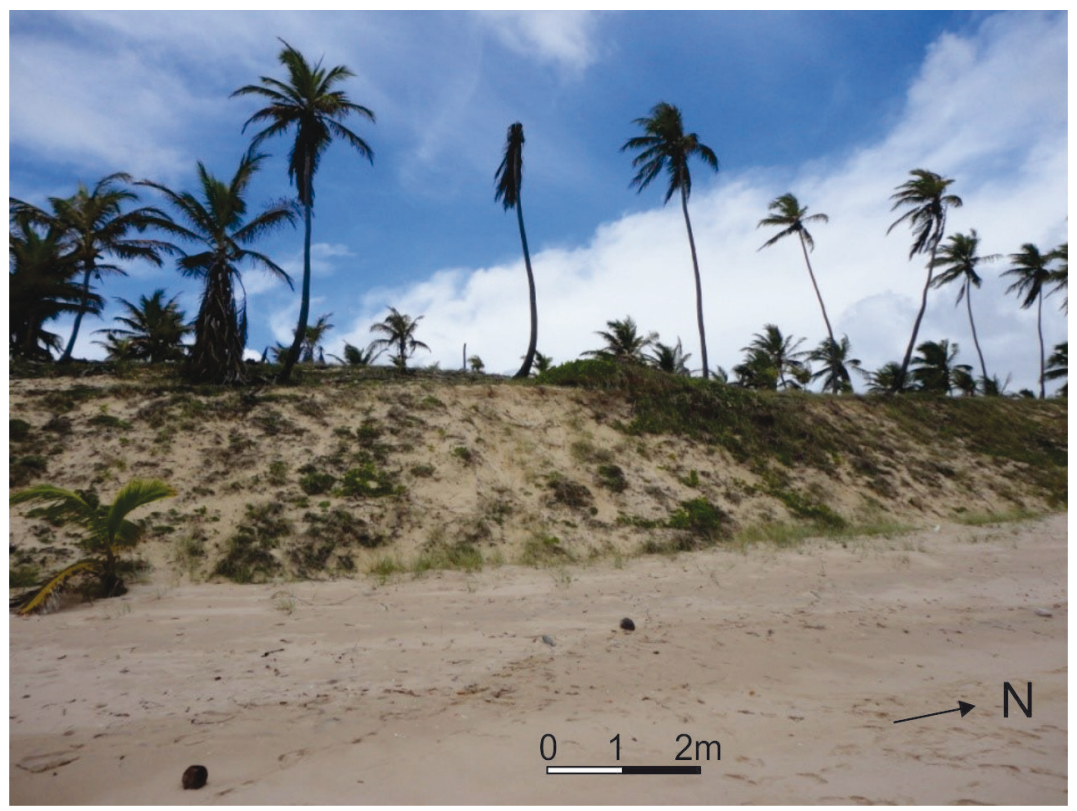

Figura 3. Cordão-duna e plantação de coqueiros ao norte do Rio Inhambupe e do povoado de Baixio.

Figure 3. Beach ridge and coconut tree crop located northwards from the Inhambupe River and the village of Baixio.

\subsection{Métodos}

A modelagem de ondas utilizou a base de dados batimétricos obtida a partir do módulo IH-DATA (BACO) do SMC-TOOLS. Este é um dos módulos do SMC-Brasil que emprega dados fornecidos pelas cartas náuticas brasileiras (IH-CANTÁBRIA, 2013).

Para a análise estatística de ondas, o SMC disponibiliza um banco de dados denominado Downscaled Ocean Waves (DOW), obtido a partir do processo de reanálise (downscaling) para águas intermediárias e rasas, a partir de dados do Global Ocean Waves. Estes dados são referentes a uma série temporal, como mencionado anteriormente, de 1948 a 2008, com resolução horária, obtida por meio de modelagem numérica em uma malha global com o programa Wave Watch III (WWIII) utilizando como forçante a reanálise atmosférica do National Centers for Environmental Prediction/ National Center for Atmospheric Research (NCEP/ NCAR) (IH-CANTÁBRIA, 2013).

As séries de estados de mar, armazenadas no banco de dados do programa em pontos (nós das malhas de propagação) denominados de "pontos DOW", são as condições de contorno para a propagação dessas séries até os pontos localizados na costa.

A análise estatística de ondas para a região marinha da área de estudo, em termos de condições médias e extremas, foi realizada para um Ponto DOW (Fig. 4), com cota batimétrica de 40,68 m, utilizando o módulo AMEVA do SMC-Brasil. Este ponto foi escolhido por ser o ponto DOW mais pro- fundo disponível no banco de dados para a área de estudo. Assim, foram geradas informações sobre altura, período e direção de ondas em condições médias e de tempestade, bem como de marés meteorológicas e astronômicas. Posteriormente, foi realizada a análise de frequência dos eventos extremos da série temporal de ondas, ajustando-se uma curva de distribuição de probabilidades GEV aos valores máximos anuais de período e altura de onda, para obter os períodos de retorno (intervalos de recorrência), respectivamente, dos períodos e das alturas de ondas de diferentes magnitudes.

A partir do pré-processo de modelagem foram identificadas as frentes-de-onda mais significativas para a região, sendo então criadas duas malhas para a propagação de ondas vindas dos quadrantes de leste (E) e de sudeste (SE), com espaçamento de $25 \times 25 \mathrm{~m}$. A partir do módulo MOPLA 3.0 do SMC-Brasil, foram realizadas as propagações destas frentes-de-onda até a linha de costa, sendo considerados 150 casos de propagação, indicados pelo programa como situações de altura e direção de ondas de ocorrência mais representativas do clima de ondas em águas intermediárias no ponto DOW escolhido, para situações de preamar, maré intermediária e baixa-mar.

Ainda utilizando o módulo MOPLA 3.0 foram elaborados sete perfis perpendiculares à linha de costa, ao longo do litoral estudado, sendo para cada um deles indicada a zona de quebra das ondas. Foram também calculados parâmetros estatísticos de ondas e marés (direção, período e altura de ondas em situações médias e de tempestade, marés meteorológicas e astronômicas) para os pontos 
localizados no início da zona de arrebentação em cada um dos perfis.

Ao longo destes perfis foi calculado o transporte de sedimentos, considerando os seguintes parâmetros do sedimento: tamanho médio (areia média $=0,4 \mathrm{~mm}$ ), peso específico (Rho-s $=2650$ $\left.\mathrm{kg} / \mathrm{m}^{3}\right)$ e porosidade $(\mathrm{n}=0,4)$. Para este cálculo foi estimada a média anual de toda a série temporal estudada, com base nas equações de Bayram et al. (2007), que supõe que o sedimento é suspenso pela ação da quebra das ondas e transportado por qualquer tipo de corrente longitudinal.

A mesma série temporal de ondas foi empregada para o cálculo da cota de inundação instantânea para os sete perfis, através da soma dos componentes das marés astronômicas e meteorológicas e do espraiamento das ondas. Novamente, foi realizada a análise de frequência de eventos meteorológicos extremos, considerando-se as cotas máximas anuais, que foram ajustadas a uma curva de distribuição GEV, obtendo-se o período de retorno das cotas de inundação segundo a sua magnitude.

\section{Resultados}

A modelagem de ondas e correntes feitas através do SMC-Brasil permitiu identificar as características de ondas e marés para a região marinha da área de estudo e modelar a sua propagação para zonas intermediárias e rasas. A partir desta modelagem foram calculados o transporte de sedimentos e a cota de inundação máxima para esse litoral.

Os dados gerados pelo SMC-Brasil para o ponto DOW escolhido (Fig. 4) indicaram que as ondas provenientes de ESE e de SE representam, respectivamente, 52 e $43 \%$ das ondas incidentes neste ponto. A altura para as ondas de ESE em condições de tempestade foi de 3,14 $\mathrm{m}$ e, para as de SE, de $3,25 \mathrm{~m}$. Para as condições medianas, a altura de onda significativa foi de $1,61 \mathrm{~m}$ para ondas provenientes de ESE e de 1,66 m para as de SE.

0 período para as condições de tempestade foi de 11,96 s para ondas provenientes de ESE e de 13,81 s para as de SE. Para as condições medianas, o período foi de $7,87 \mathrm{~s}$ para ondas provenientes de ESE e de 7,21 s para as de SE.

0 ajuste de valores extremos por máximos anuais indicou que, aproximadamente, ondas, por exemplo, com altura de 3,5 m (Fig. 5A) e período de 15,5 s (Fig. 5B), têm um período de retorno de 10 anos. Em período de retorno de 200 anos, teríamos ondas com altura de $3,8 \mathrm{~m}$ e período de $16,8 \mathrm{~s}$.

0 regime de marés na região é do tipo mesomaré semi-diurna. Os resultados mostram que o regime de oscilação do nível do mar é principalmente governado pela maré astronômica, com oscilações (para condições de meia-maré) de $\pm 1,25$ $\mathrm{m}$, enquanto as marés meteorológicas, de $\pm 0,2 \mathrm{~m}$.

O sentido resultante de propagação das ondas

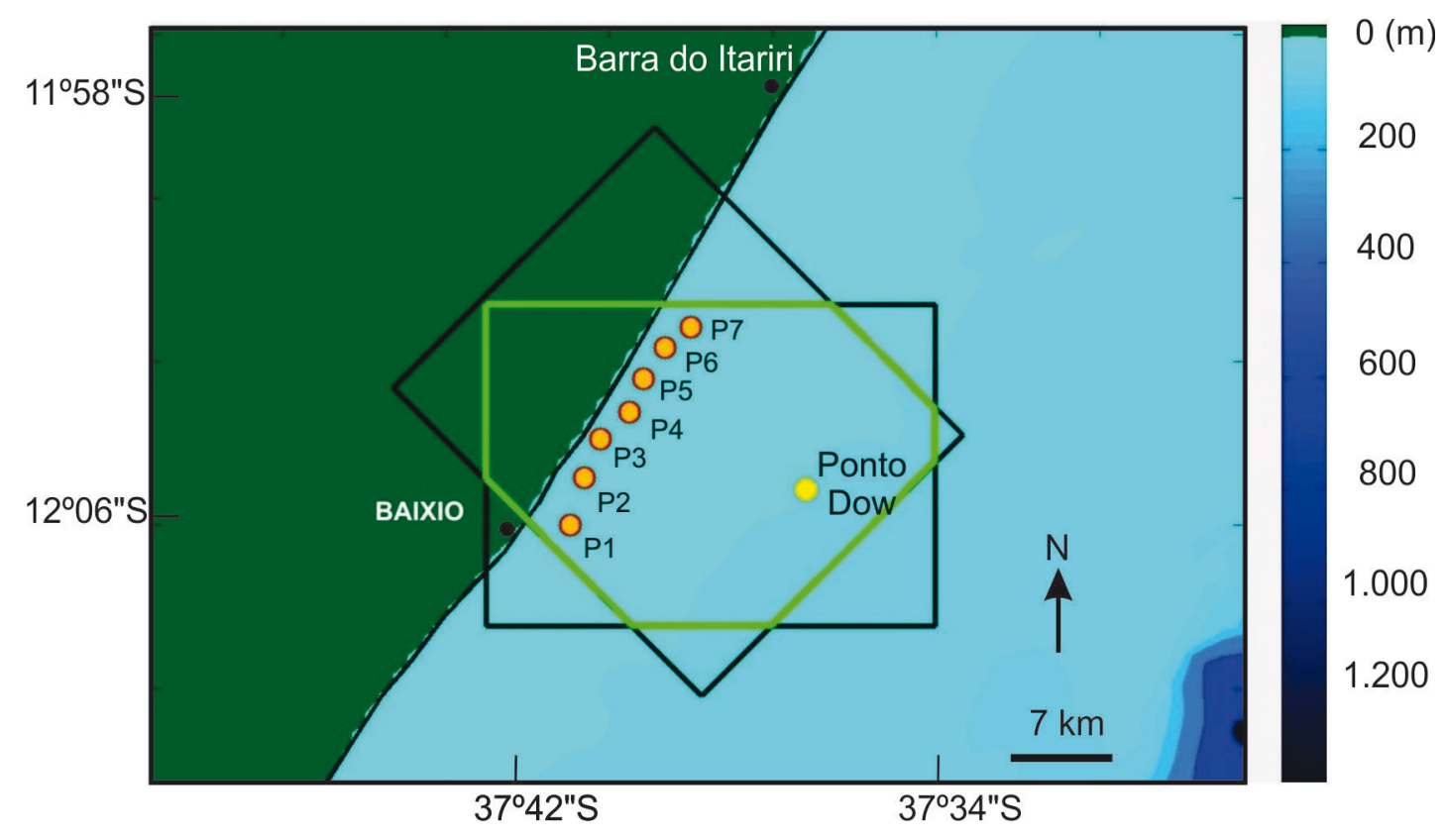

Figura 4. Localização do Ponto DOW escolhido para a análise estatística de ondas na região marinha, dos sete pontos para análise em águas rasas no início da zona de quebra dos perfis e o contorno das malhas para propagação das ondas (em preto). 0 contorno da área de interseção entre as duas malhas está marcado em verde.

Figure 4. Location of the DOW Point chosen for describing wave statistical analysis in the marine area, the position of the seven points for the shallow-water analysis at the beginning of the surf zone and the contour of the wave propagation meshes (in black). The contour of the intersection area between the two meshes is marked in green. 
é apresentado na figura 6, as quais são predominantemente oriundas de SE, alcançando alturas em torno de 1,4 m na zona de arrebentação. Os efeitos da refração são suaves, pois a direção resultante das frentes-de-onda é quase perpendicular à orientação da linha de costa (Fig. 6).

As correntes longitudinais geradas pelas ondas na zona de surfe têm um sentido predominante para NE, com uma gradativa acentuação à medida que se deslocam, com magnitudes de 0,04 a 0,05 $\mathrm{m} / \mathrm{s}$, aproximadamente. Além disso, observam-se em três pontos, equidistantemente distribuídos na zona de surfe, com espaçamento aproximado entre eles de $3 \mathrm{~km}$, a ocorrência de correntes de retorno com velocidade máxima de aproximadamente 0,029 m/s (Fig. 7). Para além da zona de surfe, as correntes geradas pelas ondas são desprezíveis.

A avaliação do clima de ondas em águas rasas, nos pontos selecionados (Fig. 4) no início da zona de arrebentação dos sete perfis estudados indicou, para condições médias (As) (Tab. 1), alturas entre 1,24 e 1,62 m e, para condições de tempestade (As12), entre 2,08 e 3,05 m. 0 ponto 1 apresentou as maiores alturas, com altura máxima em condições de tempestade de 3,05 m para ondas de SE. Contudo, este ponto apresenta o menor percentu-

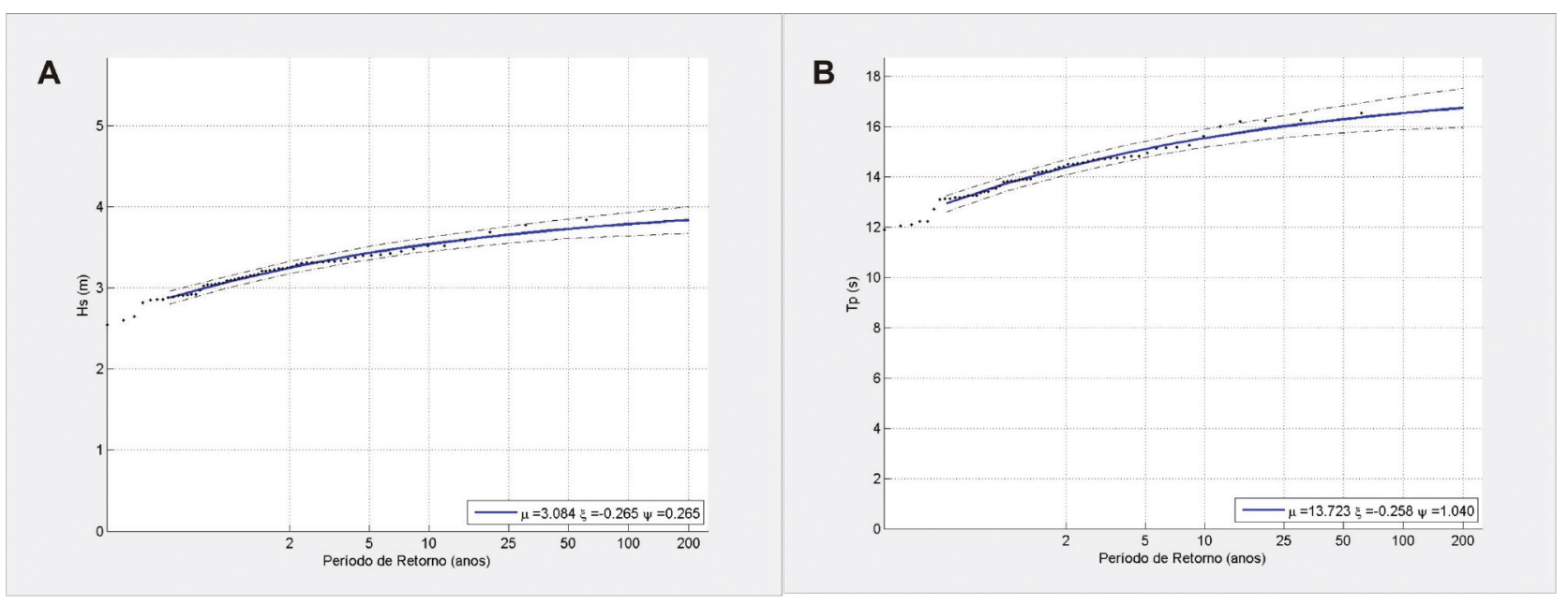

Figura 5. Ajuste de valores extremos por máximos anuais com períodos de retorno de 2, 5, 10, 25, 50, 100 e 200 anos, para a altura (A) e período das ondas (B).

Figure 5. Adjustment of extreme values per annual maximums with return periods of 2, 5, 10, 25, 50, 100 and 200 years, for wave height (A) and wave period (B).

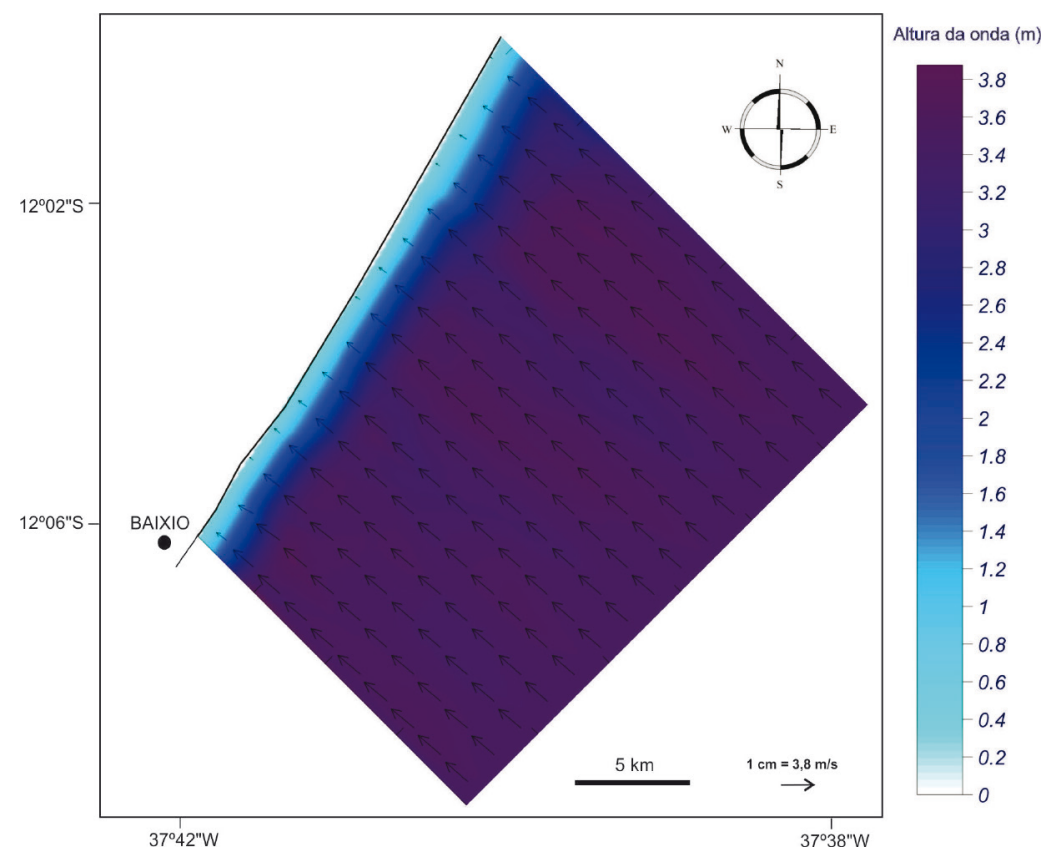

Figura 6. Sentido, velocidade de propagação e altura resultante das ondas na área de estudo. A legenda ao lado direito da figura representa a profundidade batimétrica em metros.

Figure 6. Wave direction, velocity and height propagated in the study area. The legend on the right side of the figure represents bathymetric depth in meters. 
al de ocorrência das ondas de SE dentre os pontos avaliados (47\%). No ponto 4 o percentual de incidência das ondas de SE é praticamente igual ao das ondas de ESE, com 49 e $50 \%$, respectivamente. $\mathrm{Na}$ maior parte dos demais pontos o percentual de incidência das ondas de SE é maior que o das ondas de ESE.

O sentido do transporte predominante é de SW para NE na maioria dos perfis estudados (Tab. 2). Contudo, nas proximidades de Baixio (Perfil 1) o transporte é de NE para SW, com magnitude muito baixa (Fig. 8), indicando a existência de uma zona de divergência no sentido do transporte líquido, com um aumento da intensidade do trans- porte para norte da área de estudo.

A figura 9 apresenta os valores das cotas de inundação (Ci) máximas anuais e a sua recorrência em períodos de 2, 5, 10, 25, 50, 100 e 200 anos, através do ajuste de valores extremos a uma curva de distribuição GEV, para os sete perfis. Assim, para um período de recorrência de, por exemplo, 10 anos, a partir de dados de ondas propagadas até a costa, a cota de inundação varia de 2,5 a 2,7 $\mathrm{m}$ nos perfis estudados. Com um período de recorrência de 200 anos, as cotas de inundação variam entre 2,8 e $2,9 \mathrm{~m}$.

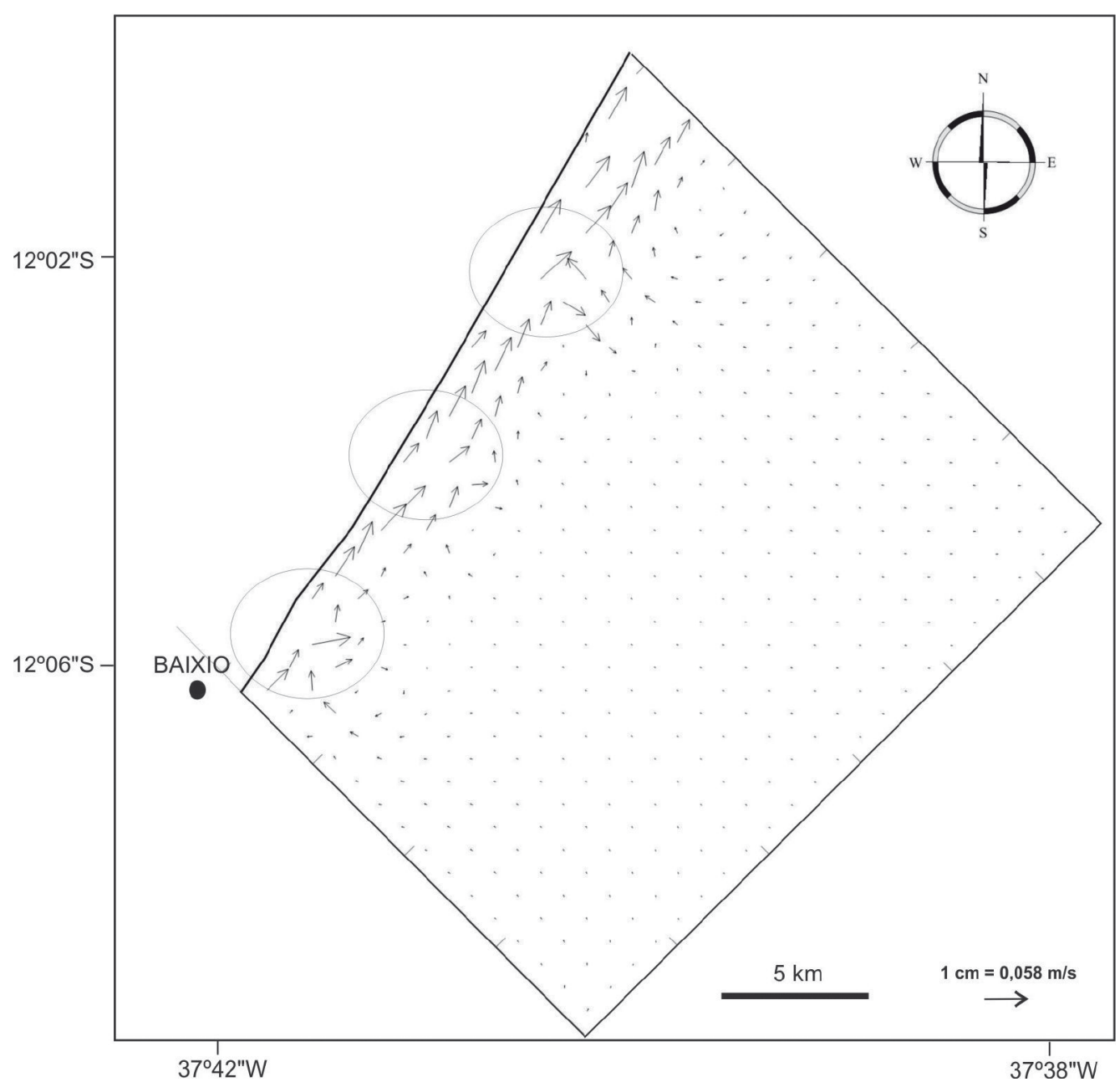

Figura 7. Sentido e magnitude das correntes geradas pelas ondas na área de estudo. Os três círculos indicam zonas com correntes de retorno.

Figure 7. Direction and magnitude of wave-induced currents in the study area. The three circles indicate areas with rip currents. 
Tabela 1. Probabilidade de ocorrência e alturas das ondas em condições médias e de tempestade para as principais frentes de onda nos sete pontos analisados ao longo da área de estudo.

Table 1. Probability of occurrence and wave heights under mean and extreme conditions for the main wave fronts in the seven points evaluated along the study area.

\begin{tabular}{|c|c|c|c|c|}
\hline Pontos & $\begin{array}{l}\text { Direções } \\
\text { principais }\end{array}$ & $\begin{array}{l}\text { Probabilidade de } \\
\text { ocorrência }\end{array}$ & Hs (m) & Hs12 (m) \\
\hline \multirow{2}{*}{01} & ESE & $52 \%$ & 1,45 & 2,85 \\
\hline & $\mathrm{SE}$ & $47 \%$ & 1,62 & 3,05 \\
\hline \multirow{2}{*}{02} & ESE & $41 \%$ & 1,33 & 2,44 \\
\hline & SE & $59 \%$ & 1,55 & 2,65 \\
\hline \multirow{2}{*}{03} & ESE & $43 \%$ & 1,38 & 2,08 \\
\hline & SE & $57 \%$ & 1,49 & 2,15 \\
\hline \multirow{2}{*}{04} & ESE & $50 \%$ & 1,32 & 2,43 \\
\hline & SE & $49 \%$ & 1,44 & 2,57 \\
\hline \multirow{2}{*}{05} & ESE & $41 \%$ & 1,38 & 2,30 \\
\hline & SE & $59 \%$ & 1,54 & 2,43 \\
\hline \multirow{2}{*}{06} & ESE & $32 \%$ & 1,28 & 2,25 \\
\hline & $\mathrm{SE}$ & $67 \%$ & 1,46 & 2,44 \\
\hline \multirow{2}{*}{07} & ESE & $28 \%$ & 1,24 & 2,31 \\
\hline & SE & $71 \%$ & 1,37 & 2,32 \\
\hline
\end{tabular}

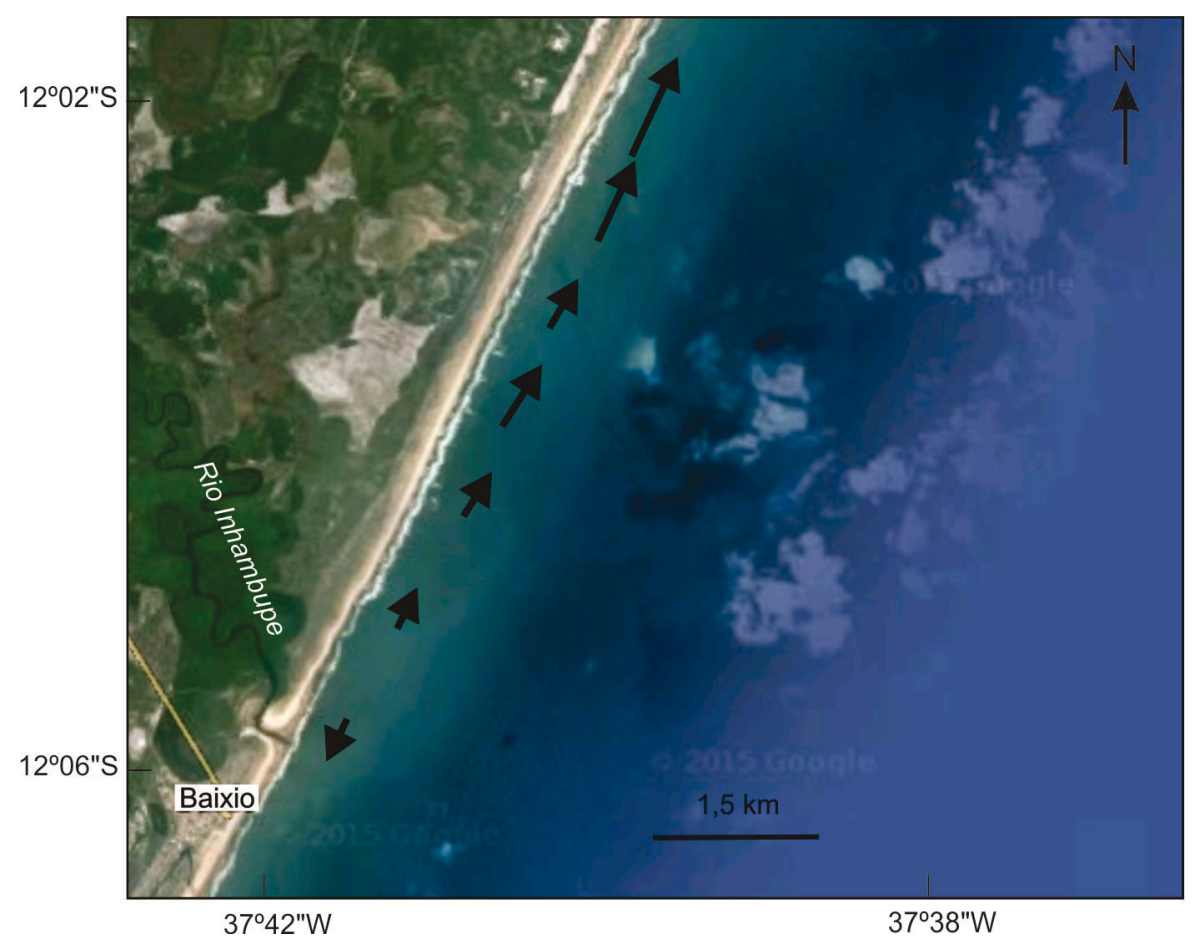

Figura 8. Sentido do transporte litorâneo líquido calculado para os sete perfis.

Figure 8. Direction of the net longshore transport calculated for the seven profiles. 
Tabela 2. Transporte litorâneo médio anual (série temporal de 1948 a 2008) positivo, negativo e líquido para os sete perfis estudados.

Table 2. Mean positive, negative and net annual longshore transport (temporal series from 1948 through 2008) for the seven studied profiles.

\begin{tabular}{c|c|c|c}
\hline Perfil & $\begin{array}{c}\text { Componente NE-SW } \\
(\mathbf{Q}+)\left(\mathbf{m}^{3}\right)\end{array}$ & $\begin{array}{c}\text { Componente } \mathbf{S W}-\mathbf{N E} \\
(\mathbf{Q})\end{array}$ & $\begin{array}{c}\text { Transporte } \\
\text { Líquido } \mathbf{( m}^{\mathbf{3}} \mathbf{)}\end{array}$ \\
\hline $\mathbf{0 1}$ & $1.758,17$ & $-1.739,19$ & 18,99 \\
\hline $\mathbf{0 2}$ & $1.002,78$ & $-1.848,41$ & $-845,63$ \\
\hline $\mathbf{0 3}$ & $1.010,33$ & $-3.045,00$ & $-2.043,68$ \\
\hline $\mathbf{0 4}$ & $1.210,93$ & $-4.617,74$ & $-3.406,81$ \\
\hline $\mathbf{0 5}$ & 431,18 & $-2.822,05$ & $-2.390,88$ \\
\hline $\mathbf{0 6}$ & 248,21 & $-4.473,20$ & $-4.224,99$ \\
\hline $\mathbf{0 7}$ & 36,04 & $-5.168,74$ & $-5.132,70$ \\
\hline
\end{tabular}

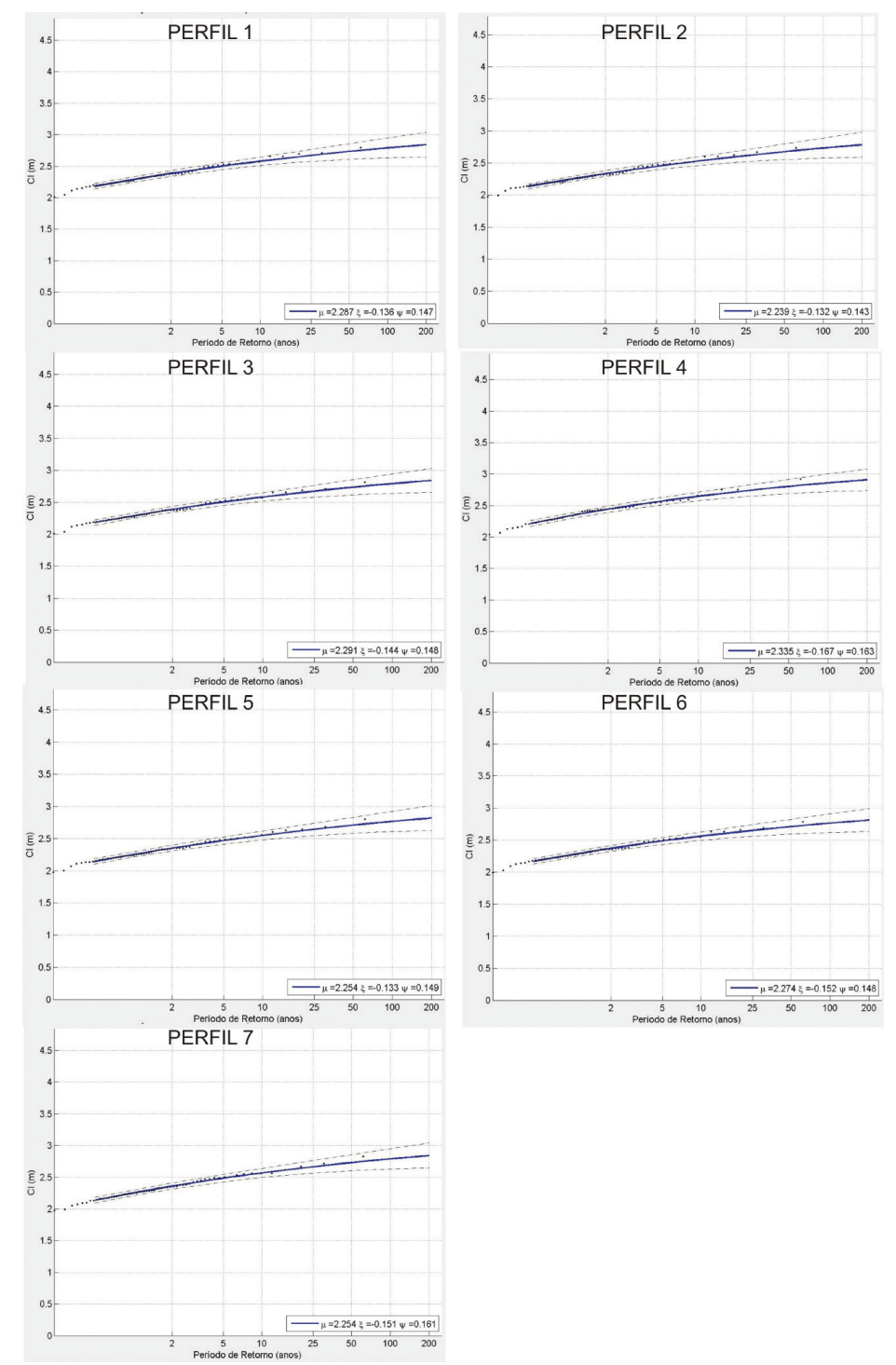

Figura 9. Cota de inundação (Ci) e a sua recorrência em períodos de 2, 5, 10, 25, 50, 100 e 200 anos, através do ajuste de valores extremos por máximos anuais, para os perfis de 1 a 7 , respectivamente representados nas figuras de 9A a 9G.

Figure 9. Inundation level (Ci) and its occurrence in periods of 2, 5, 10, 25, 50, 100 and 200 years, through the adjustment of extreme values per annual maximums for profiles 1 through 7 , respectively represented in figures $9 \mathrm{~A}$ trough $9 \mathrm{G}$. 


\section{Discussão dos resultados}

Os resultados encontrados nesta pesquisa corroboraram o processo erosivo presente na região de Baixio e representou uma oportunidade de testar e aplicar o SMC-Brasil para um trecho do litoral da Bahia.

No presente estudo, a análise do clima de ondas em águas rasas indicou a ocorrência de ondas acima de $3 \mathrm{~m}$ para condições de tempestade no início da zona de arrebentação no ponto $1 \mathrm{e}$, acima de 2,5 m, nos pontos 2 e 4 (Tab. 1). Considerando que a altura da onda é proporcional ao seu poder de ataque, isso pode representar uma maior vulnerabilidade a eventos erosivos nestes pontos.
A modelagem do transporte litorâneo utilizando o SMC-Brasil indicou a existência de uma zona de divergência no sentido do transporte líquido de sedimentos na área de estudo, como já apontado em trabalhos anteriores feitos com outros métodos de modelagem (Livramento, 2008; Bittencourt et al., 2010; Santana Neto, 2012). Isso ocorre devido a uma leve alteração na angulação da linha de costa, que favorece o transporte de norte para sul, sentido inverso do padrão geral do litoral norte da Bahia, que é em geral de sul para norte. Indicadores geomórficos de deriva, como os pontais arenosos do Rio Inhambupe, em Baixio, e do Rio Itariri, ao norte da área, também indicam essa zona de divergência no transporte litorâneo (Fig. 10).
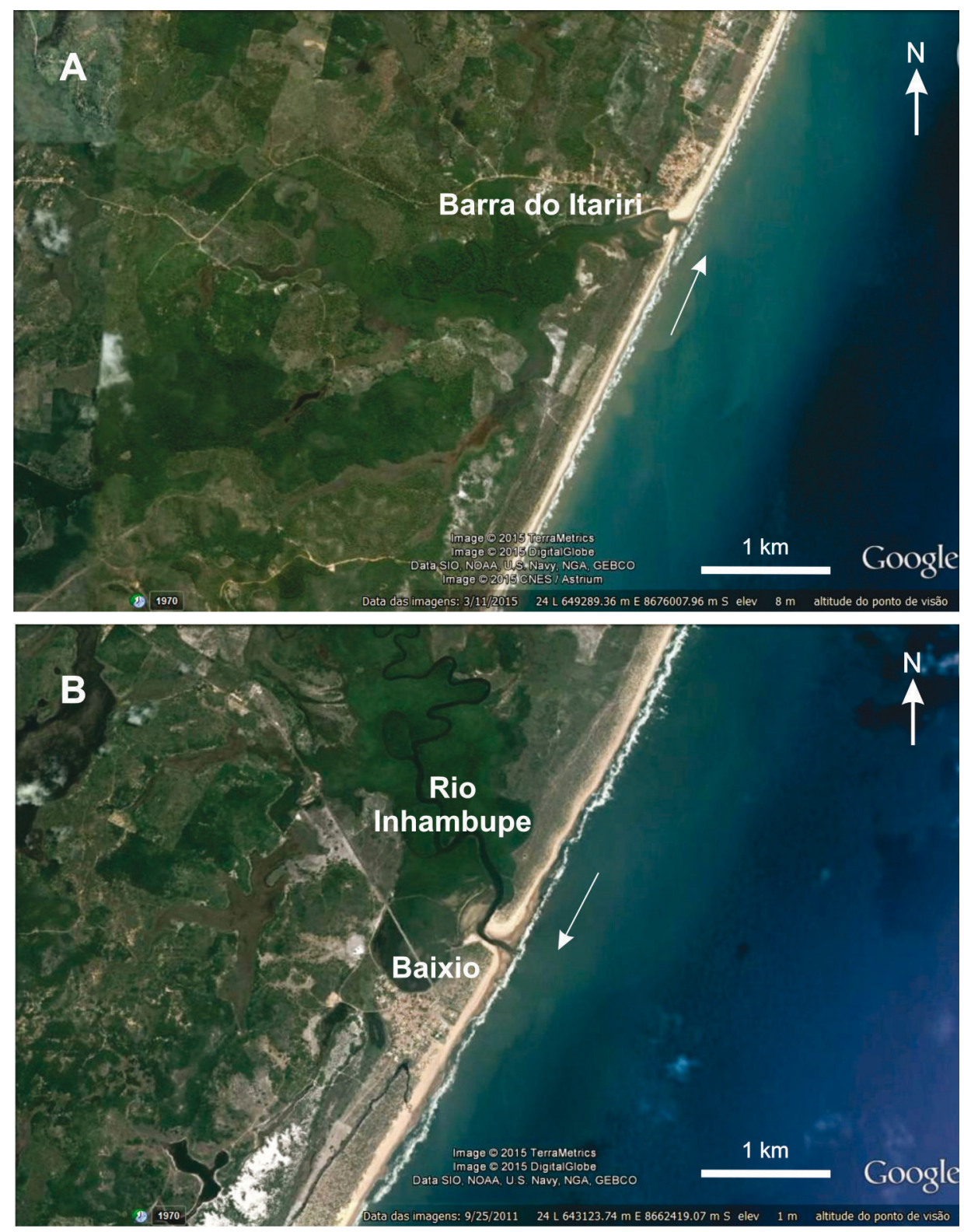

Figura 10. Indicação da deriva litorânea de sedimentos pelos pontais arenosos dos rios Itariri (A) e Inhambupe (B) (Fonte: Google, 2015).

Figure 10. Indication of the longshore drift by sandy spits or the Itariri (A) and Inhambupe (B) rivers (Source: Google, 2015). 
De um modo geral, ocorre um aumento na intensidade do transporte de sedimentos para o nordeste da área de estudo (Fig. 8), o que está de acordo, por exemplo, com a modelagem feita por Santana Neto (2012). Neste sentido, segundo este autor, ocorre um aumento progressivo, nas praias, de ocorrência de lixo marinho a partir de Baixio, para norte.

A cota de inundação é determinada pela ação conjunta das marés astronômica e meteorológica com o espraiamento das ondas. Em geral, para a área de estudo, variou em torno de 2 a 2,2 m. Contudo, em período de recorrência acima de 5 anos, esses valores aumentar e chegar a quase $3 \mathrm{~m}$. A determinação deste nível e de sua probabilidade de ocorrência e período de retorno, em anos, é fundamental para a gestão costeira, especialmente no planejamento de medidas preventivas contra inundações, bem como para a recuperação de ecossistemas costeiros (Rodrigues, 2014). Apesar dessa análise não ser o foco deste estudo, deve-se considerar que a ocupação dos terraços arenosos, presentes na Vila de Baixio, dificultando os processos de infiltração, e as alterações da dinâmica hídrica natural com os empreendimentos instalados nas zonas úmidas tornam esta região ainda mais vulnerável à inundação, especialmente nas zonas mais baixas próximas à foz do Rio Inhambupe. A presença do cordão-duna, que bordeja boa parte do litoral em estudo, protege a região à sua retaguarda. Contudo, a existente retirada da vegetação e a alteração na morfologia destes cordões diminuem a sua proteção natural, aumentando o risco frente a possíveis eventos extremos.

Por fim, não se pode desconsiderar, tento em vista a vulnerabilidade deste trecho costeiro, as previsões de subida do nível do mar e de aumento da frequência e magnitude das tempestades tropicais (Muehe \& Neves, 1995; Zhang et al., 1997; Walsh et al., 2004), com seus potenciais impactos na linha de costa (SCOR, 1991; Neves \& Muehe, 1995; Masselink \& Hughes, 2003).

\section{Conclusões}

Através da modelagem de ondas e correntes costeiras, utilizando o SMC-Brasil, para o litoral entre os povoados de Baixio e de Barra do Itariri, foi possível gerar modelos de propagação de ondas, de transporte sedimentar e de cota de inundação máxima para este litoral. A análise estatística de ondas realizada para águas intermediárias indicou um predomínio das ondas vindas de ESE e SE, com alturas variando de 1,61 a 3,25 m e períodos variando de 7,87 a 13,81 s. A modelagem do clima de ondas em águas rasas, analisada em sete pontos do litoral, indicou uma predominância das ondas de SE, alcançando alturas em torno de 1,4 m na zona de arrebentação, e a ocorrência de três zonas de correntes de retorno.

Foi possível também identificar uma zona de divergência no sentido do transporte líquido de sedimentos, nas proximidades de Baixio, já apontada em trabalhos anteriores, servindo assim para validar esta ferramenta de modelagem. Além disso, o cálculo das taxas médias anuais de transporte longitudinal indicou um aumento no percentual de incidência das ondas de SE seguindo para norte da área.

A estimativa da cota de inundação, baseada na análise de frequência dos valores máximos anuais, com períodos de recorrência de 2, 5, 10, 25, 50, 100 e 200 anos, indicou, em geral, níveis entre 2,5 e 3,0 m para os sete perfis.

A combinação de eventos de tempestades com marés meteorológicas positivas, marés astronômicas de sizígia e valores extremos de espraiamento de onda, pode resultar em situações de alto risco para ecossistemas e propriedades. Essa situação poderá gerar grandes impactos para o litoral estudado, que atualmente já apresenta uma alta vulnerabilidade à erosão costeira. Recomenda-se, portanto, que seja evitada a ocupação por construções fixas na região litorânea mais próxima à linha de costa, respeitando-se no mínimo o limite de 200 $\mathrm{m}$ da linha de preamar de sizígia.

Agradecimentos - I.R. Silva e A.C.S.P. Bittencourt agradecem ao $\mathrm{CNPq}$ (Conselho Nacional de Desenvolvimento Científico e Tecnológico) pela bolsa de Produtividade em Pesquisa e G. Fernandino por sua bolsa de Doutorado.

\section{Referências}

Almeida, L.R. 2013. Estudio de dinámica litoral y evolución de la zona sur de la Playa de Piçarras (Santa Catarina/Brasil). Santander, 263p. Dissertação de Mestrado, Universidade de Cantabria.

Almeida, L.R., Amaro, V.E., Marcelino, A.M.T. \& Scudelari, A.C. 2015. Avaliação do clima de ondas da praia de Ponta Negra (RN, Brasil) através do uso do SMC-Brasil e sua contribuição à gestão costeira. Revista de Gestão Costeira Integrada, 15(2): 135-151.

Bayram, A., Larson, M. \& Hanson, H. 2007. A new formula for the total longshore sediment transport rate. Coastal Engineering, 540(9): 700-710. Bittencourt, A.C.S.P., Livramento, F.C., Dominguez, 
J.M.L. \& Silva, I.R. 2010. Tendências de longo prazo à erosão costeira num cenário perspectivo de ocupação humana: Litoral Norte do Estado da Bahia. Revista Brasileira de Geociências, 40: 125-137.

Calliari, L.J., Guedes, R.M.C., Pereira, P.S., Lélis, R.F., Antiqueira, J.A., Figueiredo, S.A. \& Baz, J. 2010. Perigos e Riscos Associados a Processos Costeiros no Sul do Litoral do Brasil. Brazilian Journal of Aquatic Science and Technology, 14(1): 51-63.

Camus, P., Mendez, F.J, Medina, R., Tomas, A. \& Izaguirre, C. 2013. High resolution downscaled ocean waves (DOW) reanalysis in coastal areas. Coastal Engineering, 72: 56-68.

Casagrande, A., Silva Júnior, P. \& Mendonça, F. 2011. Mudanças climáticas e aquecimento global: controvérsias, incertezas e a divulgação científica. Revista Brasileira de Climatologia, 8: 30-44.

Diniz, I.N. \& Resende, R. 2014. Cartilha: Mudança Global do Clima. Pinheiros, Iniciativa Verde, 43 p.

Dominguez, J.M.L. \& Bittencourt, A.C.S.P. 1994. Utilização de padrões de sedimentação costeira como indicadores paleoclimáticos naturais (proxies). Revista Brasileira de Geociências, 24(1): 3-12.

Dominguez, J.M.L., Andrade, A.C.S., Almeida, A.B. \& Bittencourt, A.C.S.P. 2009. The Holocene Barrier Strandplains of the State of Bahia. In: Dillenburg S.R. \& Hesp, P.A. (Eds.), Geology and Geomorphology of Holocene Coastal Barriers of Brazil. Lecture Notes in Earth Sciences. Berlin, Springer-Verlag, 288p.

Douglas, B.C. \& Peltier, W.R. 2002. The puzzle of global sea-level rise. Physics Today: 35-40.

Egler, C.A.G. 1996. Risco Ambiental como Critério de Gestão do Território: uma Aplicação à Zona Costeira Brasileira. Revista Território, 1(1): 3141.

Fitzgerald, D.M., Fenster, M.S., Argow, B.A. \& Buynevich, I.V. 2008. Coastal Impacts Due to Sea-Level Rise. Annual Review of Earth and Planetary Sciences, 36: 601-647.

IH-CANTÁBRIA. 2013. Ondas: Documento Temático. IHCANTABRIA, Universidad de Cantabria/ UFSC/IO-USP, 155p.

IPCC. Intergovernmental Panel on Climate Change. 2007. Climate Change 2007: The Physical Science Basis. Contribution of Working Group I to the Fourth Assessment Report of the Intergovernmental Panel on Climate Change [Solomon, S., D. Qin, M. Manning, Z. Chen, M. Marquis, K. B. Averyt, M. Tignor, H. L. Miller (Orgs.)]. Cambridge, Cambridge University Press, 996p.

Leão, Z.M.A.N. \& Kikuchi, R.K.P. 1999. The Bahian
Coral Reefs - from 7000 years BP to 2000 years AD. Ciência \& Cultura, 51: 262-273.

Livramento, F.C. 2008. Padrões de dispersão de sedimentos ao longo do litoral norte do Estado da Bahia: subsídios para o gerenciamento costeiro. Salvador, 51p. Monografia de Conclusão de Curso, Curso de Geologia, Instituto de Geociências, Universidade Federal da Bahia.

Livramento, F.C. 2013. O Papel da Herança Geológica, das Ondas, e da Deriva Litorânea no Condicionamento das Características Morfodinâmicas e Texturais das Praias da Costa dos Coqueiros/Ba. Salvador, 51p. Dissertação de Mestrado, Curso de Pós-Graduação em Geologia, Instituto de Geociências, Universidade Federal da Bahia.

Luca, C.B. 2011. Implementação de ferramentas numéricas e bases de dados no SMC-Brasil e sua aplicação no estudo piloto da praia de Massaguaçu-Brasil. Santander, 213p. Dissertação de Mestrado, Universidade de Cantabria.

Marengo, J.A. 2008. Águas e mudanças climáticas. Estudos Avançados, 22(63): 84-96.

Martin, L., Bittencourt, A.C.S.P., Vilas Boas, G.S. \& Flexor, J.M. 1980a. Texto Explicativo para o Mapa Geológico do Quaternário Costeiro do Estado da Bahia. Salvador, CPM/SME, $20 \mathrm{p}$.

Martin, L., Bittencourt, A.C.S.P., Vilas Boas, G.S. \& Flexor, J.M. 1980b. Mapa Geológico do Quaternário Costeiro do Estado da Bahia, escala 1:250.000. Salvador, CPM/SME.

Masselink G. \& Hughes M.G. 2003. Introduction to Coastal Processes and Geomorphology. London, Hodder Arnold, 354p.

Miranda, L. de A. \& Araújo, R. de S. 2014. Mudanças climáticas: análise de dois pontos de vista. Revista Perspectivas On Line: Ciências Humanas e Sociais Aplicadas, 9(4): 47-55.

Molion, L.C.B. 2008. Aquecimento global: uma visão crítica. Revista Brasileira de Climatologia, 3/4: 7-24.

Muehe, D. \& Neves, C.F. 1995. The implication of sea level rise on the Brazilian coast: a preliminary assessment. Journal of Coastal Research (SI), 14: 54-78.

Neves C.F. \& Muehe D. 1995. Potential Impacts of Sea-level Rise on the Metropolitan Region of Recife, Brazil. Journal of Coastal Research (SI), 14:116-131

Nicholls, R.J. \& Leatherman, S.P. 1995. The implications of accelerated sea-level rise for developing countries: A discussion. Journal of Coastal. Research (SI), 14: 303-323.

Nicholls, R.J. \& Cazenave, A. 2010. Sea-level rise and its impact on coastal zones. Science, 328: 1517-1520. 
Oliveira, M.J. de \& Vecchia F. 2009. A controvérsia das mudanças climáticas e do aquecimento global antropogênico: consenso científico ou interesse político? Revista Forum Ambiental da Alta Paulista, 5: 946-962.

Pereira, C. \& Coelho, C. 2013. Mapas de Risco das Zonas Costeiras por Efeito da Ação Energética do Mar. Revista de Gestão Costeira Integrada, 13(1): 27-43.

Ribeiro, W.C. 2008. Impactos das mudanças climáticas em cidades no Brasil. Parcerias Estratégicas, 27: 297-321.

Rodrigues, T.K. 2014. Impacto das Mudanças Climáticas na Zona Costeira do Município de Aracaju-SE: Estudo de Caso Utilizando o SMC (Sistema de Modelagem Costeira). Salvador, 221p. Tese de Doutorado, Programa de Pós-graduação em Geologia, Instituto de Geociências, Universidade Federal Bahia.

Santana Neto, S.P. 2012. Padrão de distribuição do lixo marinho e sua interação com a dinâmica de ondas e deriva litorânea na Costa dos Coqueiros, Bahia. Salvador, 99p. Dissertação de Mestrado, Programa de Pós-graduação em Geologia, Instituto de Geociências, Universidade Federal Bahia.

SCOR. Scientific Committee on Ocean Research Working Group, 89. 1991. The Response of bea- ches to sea-level changes: a review of predictive models. Journal of Coastal Research, 7: 895-921. Silva, I.R., Bittencourt, A.C.S.P., Dias, J.A\& Souza Filho, J.R. 2012. Qualidade recreacional e capacidade de carga das praias do litoral norte do estado da Bahia, Brasil. Gestão Costeira Integrada, 12(2): 131-146.

Suguio, K. \& Nogueira, A.C.R. 1999. Revisão Crítica dos Conhecimentos Geológicos sobre a Formação (Ou Grupo?) Barreiras do Neógeno e o seu Possível Significado como Testemunho de Alguns Eventos Geológicos Mundiais. Geociências, 18: 461-479.

Tronis, A.A. 2004. Is global warming injecting randomness into the climate system? EOS Transaction American Geophysics Union, 85(38): 361364.

Walsh, K.J.E., Church, H.B.J., Pittock A.B., Innes, K.L., Jackett, D.R. \& Dougall, T.J. 2004. Using Sea Level Rise Projection for Urban Planning in Australian. Journal of Coastal Research, 20: 586-598.

Wright, L.D. \& Short, A.D. 1984. Morphodynamics of beaches and surf zones in Australia. In: Komar P.D. (Ed.). Handbook of Coastal Process and Erosion. Boca Raton, CRC Press, 66p.

Zhang K., Douglas B.C. \& Leatherman S.P. 1997. East Coast Storm Surges Provide Unique Climate Record. EOS, 78: 389-397. 